\title{
Aspect Ratio Considerations for Flat Bottom Hole Defects in Active Thermography
}

\author{
by M. Frendberg Beemer and S. Shepard
}

Thermal Wave Imaging, Inc., 845 Livernois, Ferndale, MI 48220, USA, mfrendberg@thermalwave.com

\begin{abstract}
Flaw detection capability of a Thermographic NDT system is often characterized using machined Flat-Bottom Holes $(\mathrm{FBH})$ to simulate defects. For large diameter to depth (aspect) ratios, the $\mathrm{FBH}$ is typically indicated by the emergence of a localized hot spot in the IR image sequence. The FBH initially behaves identically to an extended adiabatic interface, allowing the center point depth to be determined using a one-dimensional model, before three-dimensional effects become dominant. However, for $\mathrm{FBH}$ with smaller aspect ratios, three-dimensional effects become dominant increasingly early, disrupting depth measurement using one-dimensional techniques. The dependence of these three-dimensional effects on aspect ratio is evident in the first and second logarithmic derivatives obtained through Thermographic Signal Reconstruction ${ }^{\circledR}$ $\left(\mathrm{TSR}^{\circledR}\right)$. In both numerical modeling and experimental results, the maximum values of the logarithmic derivatives indicate the transition between one-dimensional adiabatic behavior and the low aspect ratio regime, which occurs at an aspect ratio of approximately 9. At aspect ratios below 9, the one-dimensional depth measurement accuracy decreases proportionally with aspect ratio and the composition of a flaw cannot be reliably determined from a single pixel.
\end{abstract}

\section{Introduction}

In its simplest form, active thermographic flaw detection is based on visual identification of contrast between a flaw and local intact regions in an IR image sequence of the surface temperature as the sample responds to transient thermal stimulation [1]. The detectability of a flaw depends on numerous extrinsic factors including IR camera sensitivity, speed and resolution, excitation energy, sample surface condition and presence of background radiation or noise sources [2,3]. However, the fundamental indicators of detectability are the flaw diameter, depth and the degree to which the composition of the flaw and surrounding matrix differ. Thus, an ideal flaw is a void embedded in a solid where the flaw diameter is significantly greater than its depth, so that the flaw presents an adiabatic interface that completely obstructs the flow of heat from the excited surface. In practice, an ideal flaw is often simulated using a Flat Bottom Hole $(\mathrm{FBH})$ machined into a plate, with the understanding that this is a "best case" scenario for detectability. Large adiabatic flaws (with respect to their depth) may be detectable in the unprocessed IR image sequence; smaller flaws may require additional signal processing or considerably more excitation energy [4]. Flaw depth may be estimated using image contrast, or measured more accurately using the pixel time history signal [5].

A subsurface flaw can be described as a trap formed by the sample surfaces and the flaw, which obstructs the flow of heat, and the circumferential boundary surface between them, through which trapped heat escapes (Fig. 1). Detectability, i.e. the contrast between the flaw and surrounding area, is related to the ratio of the blockage and leakage surfaces areas, e.g. for a cylindrical hole with depth $d$, diameter $D$ and radius $r$

$$
\text { Contrast } \propto \frac{\text { Blocking Surface }}{\text { Leakage Surface }}=\frac{\pi r^{2}}{2 \pi r d}=\frac{D}{4 d} \text {. }
$$

The relationship between blocking and leakage surfaces is characterized by the ratio of diameter to depth (aspect ratio).

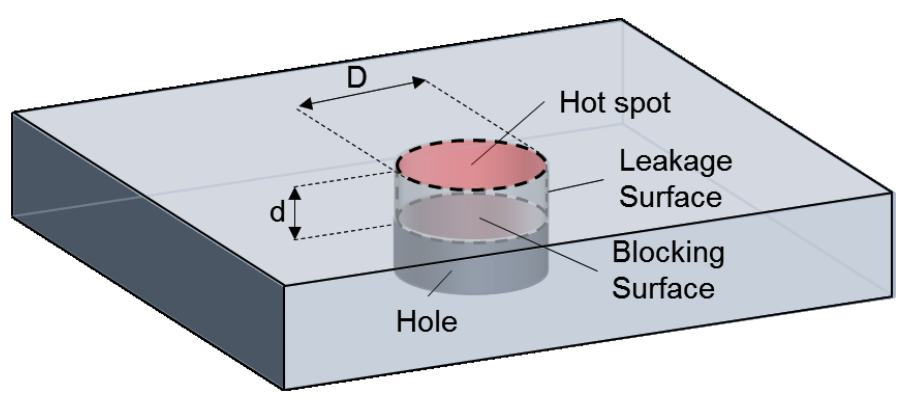

Fig. 1. Heat is trapped by the blockage and leakage surfaces of an $\mathrm{FBH}$. 


\subsection{1/qirt.2016.005}

For quantitative evaluation, the early regime of the FBH time history is often treated as identical to that of a onedimensional slab with adiabatic boundary conditions, so that the hole depth (or slab thickness), L, can be calculated using the thermal diffusivity of the host material, $\alpha$, and the characteristic time $t^{*}$, using the expression

$$
L=\sqrt{\pi \alpha \mathrm{t}^{*}}
$$

where $t^{*}$ may be determined using the intersection of the slope 0 and -0.5 asymptotes in the logarithmic temperature-time plot [6], or by the first derivative inflection point or the second derivative maximum (Fig. 2) obtained using the Thermographic Signal Reconstruction $\left(\mathrm{TSR}^{\circledR}\right)$ method. [7]

\section{Adiabatic FBH Signal Characteristics}

The surface of a semi-infinite slab heated with a uniform, instantaneous pulse cools according to one-dimensional thermal diffusion, indicated by a straight-line logarithmic temperature vs. time behavior with slope $=-0.5$. The presence of an adiabatic flaw obstructs heat conduction and the local cooling behavior deviates from straight line behavior and transitions to a quasi-steady state (slope 0 ) at time $t^{*}$, similar to the behavior of an adiabatic slab of thickness $d$ (Fig. 2). As the area surrounding the flaw cools, heat trapped by the flaw diffuses laterally toward the cooler region and three-dimensional heat conduction becomes increasingly predominant, causing the cooling behavior to break from that of the adiabatic slab.

Similar behavior is also observed in both the first and second derivatives (Fig. 2). As three-dimensional diffusion becomes the primary cooling mechanism, the adiabatic flaw signal diverges from that of the adiabatic slab, becoming a negative peak in the first derivative, or a negative peak and a final positive peak in the second derivative. The timing and amplitudes of these derivative extrema can vary depending on host material, flaw depth, and flaw diameter, but their polarities will remain the same [8]. It is important to note that a flaw is only exhibiting true adiabatic behavior while its first derivative is 0 (i.e. it is in a quasi-steady state).

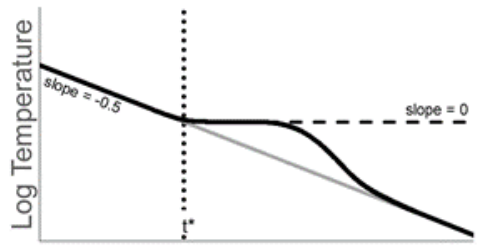

Log Time

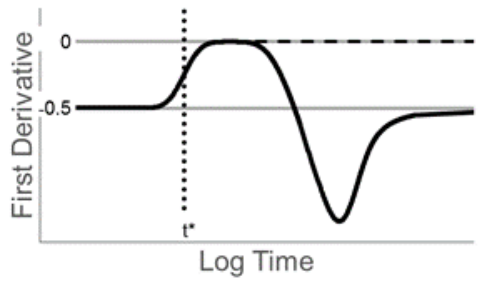

Log Time

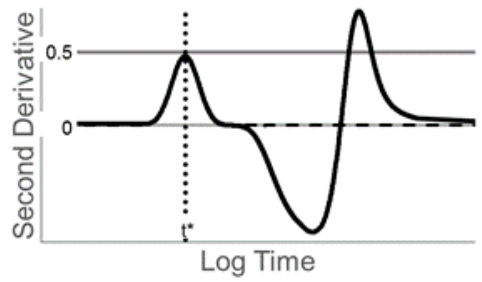

Log Time

Fig. 2. Dimensionless time-temperature response (left), first derivative (middle) and second derivative (right) to flash heating of an infinitely thick solid containing an adiabatic FBH with aspect ratio 15.

\section{Numerical Modeling}

Numerical models of $\mathrm{FBH}$ center-point temperatures in a Delrin ${ }^{\circledR}$ slab with aspect ratios spanning the range from 0.25 to 30 (Table 1) were created using a commercial finite element simulation package (COMSOL Multiphysics ${ }^{\circledR}$ ). A 2D, axisymmetric model, stimulated with a $5 \mathrm{msec}$ heat pulse of $100,000 \mathrm{~W} / \mathrm{m}^{\wedge} 2$, was monitored for $2,400 \mathrm{~s}$. Results were calculated using the implicit backward Euler technique on a free triangular mesh containing more than 900 domain elements and 400 boundary elements (the precise number of elements varied by model) with time steps growing dynamically from $1 \mathrm{x}$ e-20 s to $1 \mathrm{~s}$.

Table 1. Aspect ratio, depth, and diameter of FBH used in numerical modeling.

\begin{tabular}{|c|c|c|c|c|c|c|c|c|c|c|c|c|c|c|c|c|c|c|}
\hline tio & .25 & 0.5 & .75 & 1 & 2 & 3 & 4 & 5 & 6 & 7 & 8 & 9 & 10 & 15 & 20 & 25 & 30 & 40 \\
\hline & 2.54 & 54 & 54 & 54 & 2.54 & ( & 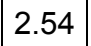 & . & & 2.54 & | & & 5 & 2.0. & 2.54 & (1). & .54 & 2.0 \\
\hline & 10.16 & 5.08 & 3.387 & 2.54 & 1.27 & 0.847 & S & | & 0 & $0.363 \mid$ & 318 & 204 & 0.254 & 0.169 & 0.127 & 0.104 & 0.085 & 0.064 \\
\hline
\end{tabular}

In the resulting temperature vs. time and first derivative data (Fig. 3), responses initially mirror the respective adiabatic slab response. The time at which the FBH signal diverges from that of the adiabatic slab increases with aspect ratio. 

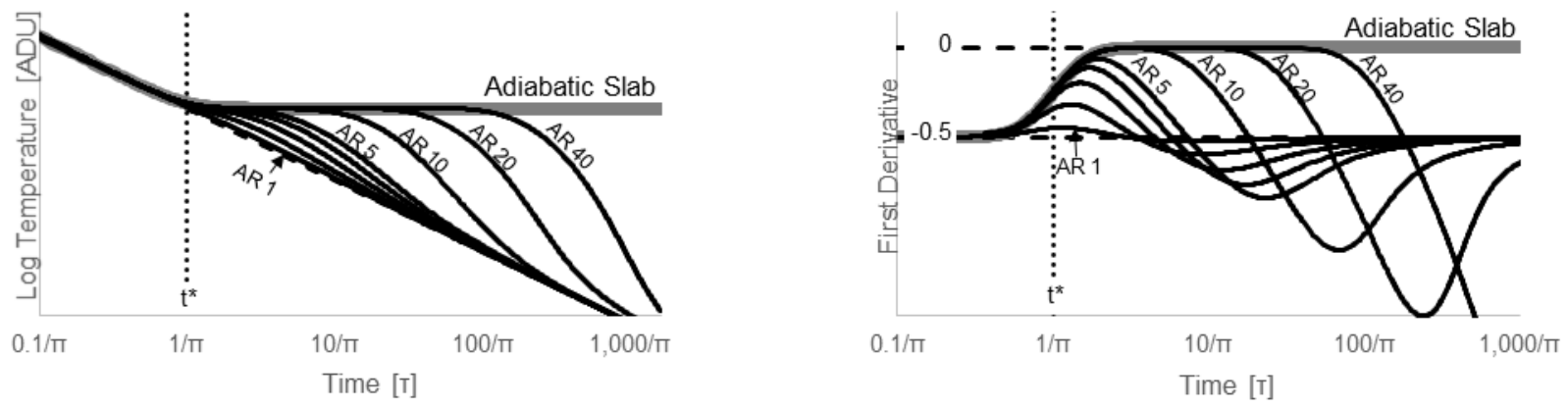

Fig. 3. Model results showing dimensionless time vs temperature response (left) and its first derivative (right) of FBH with various aspect ratios in Delrin ${ }^{\circledR}$. The aspect ratios displayed are 1, 2, 3, 4, 5, 10, 20, and 40.

\section{Experiment}

A $1.27 \mathrm{~cm}$ steel slab (Table 2) and a $1.27 \mathrm{~cm}$ stainless steel slab (Table 3), both containing programmed FBH, were evaluated with a commercial flash thermography system (EchoTherm ${ }^{\circledR}$, Thermal Wave Imaging, Inc). The system comprised an InSb IR camera, two linear xenon flashtubes housed in individual reflectors and a reflective hood, all positioned $0.36 \mathrm{~m}$ from the sample. The camera was operated at $300 \mathrm{~Hz}$ with $320 \times 256$ resolution (steel) and $40 \mathrm{~Hz}$ with $640 \times 512$ resolution (stainless steel). Each lamp was powered by a $5 \mathrm{~kJ}$ capacitor bank capable of truncating the flash to create a $0.2 \mathrm{msec}$ duration rectangular pulse, eliminating the long tail characteristics of photographic flash lamps. Data was captured for 4.46 seconds (steel) and 44.80 seconds (stainless steel), and processed using TSR to generate first and second derivatives for each pixel. One additional flat bottom hole in the stainless steel sample was ignored due to a fabrication error.

Table 2. FBH in $1.27 \mathrm{~cm}$ steel description.

\begin{tabular}{|l|l|l|l|l|l|l|l|l|l|l|}
\hline Diameter [cm] & 5.100 & 3.820 & 2.553 & 2.560 & 1.608 & 1.963 & 1.943 & 1.288 & 1.275 & 0.953 \\
\hline Depth [cm] & 0.147 & 0.170 & 0.173 & 0.246 & 0.239 & 0.226 & 0.356 & 0.325 & 0.533 & 0.439 \\
\hline Aspect Ratio & 34.6 & 22.4 & 14.8 & 10.4 & 6.7 & 8.7 & 5.5 & 4.0 & 2.4 & 2.2 \\
\hline
\end{tabular}

Table 3. FBH in $1.27 \mathrm{~cm}$ stainless steel description.

\begin{tabular}{|l|l|l|l|l|l|l|l|l|}
\hline Diameter [cm] & 2.565 & 1.905 & 2.565 & 1.93 & 1.32 & 2.565 & 1.956 & 1.321 \\
\hline Depth [cm] & 0.117 & 0.122 & 0.300 & 0.320 & 0.323 & 0.531 & 0.536 & 0.518 \\
\hline Aspect Ratio & 22.0 & 15.6 & 8.6 & 6.0 & 4.1 & 4.8 & 3.6 & 2.5 \\
\hline
\end{tabular}

\section{Results}

Data from a separate through transmission experiment was used to calculate the thermal diffusivity of each sample using the Parker method [9], resulting in values of $1.348 \mathrm{e}-5 \mathrm{~m}^{2} / \mathrm{sec}$ (steel) and $4.065 \mathrm{e}-6 \mathrm{~m}^{2} / \mathrm{sec}$ (stainless steel). Incident energy values were calculated independently for each sample. Experimental results were displayed on a dimensionless scale and compared to numerical modeling results. Both the experimental steel and stainless steel results show a strong correlation with model results from a FBH with an approximately equivalent aspect ratio (Fig. 4). Divergence occurs early in the sequence, the result of using a non-instantaneous heat pulse in the experiment, and late in the sequence, due to the interruption of one-dimensional diffusion when the incident heat reaches the back wall of the respective slab. 

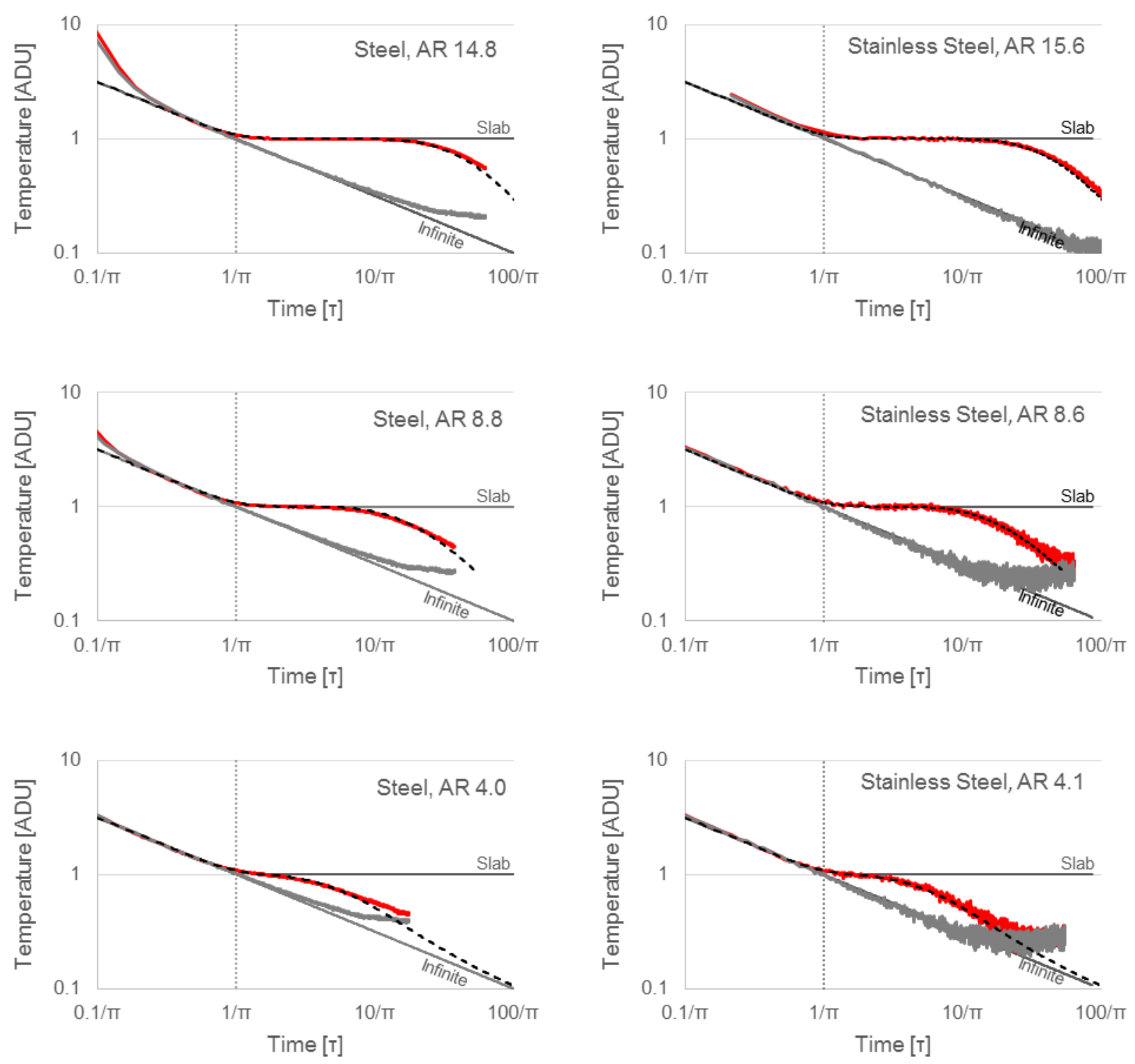

Fig. 4. Experimental FBH center-point signals (red) and background signals (gray) compared to model data (dashed black) for FBH with approximate aspect ratios of 15 (top), 9 (middle), and 4 (bottom) in steel (left) and stainless steel (right).

\section{Discussion}

Conventional FBH depth measurements described by Eq. (2) are based on a one-dimensional model which assumes that the flaw exhibits true adiabatic behavior, i.e. the logarithmic first derivative of the pixel time history reaches the adiabatic maximum amplitude of 0 , as shown in Fig. 2. However, in both modeling and experimental results (Fig. 5), it is apparent that the first derivative does not reach its full adiabatic maximum value for smaller aspect ratios, in which case the one-dimensional model does not apply.

The relationship between first derivative peak amplitude and aspect ratio is obtained by a least squares fit to the model peak amplitudes: 


\subsection{1/qirt.2016.005}

$$
1 D P A=\frac{1}{4}\left(\operatorname{erf}\left(\frac{\ln (A R)-\ln (\mu)}{\sqrt{2} * \sigma}\right)-1\right)
$$

where $\mu=2.451$ and $\sigma=0.636$. Using Eq. (3) and a first derivative maximum measurement error of 0.01 , the upper limit of the low aspect ratio regime is approximately 9 . The experimental first derivative peak amplitudes follow the dependence on aspect ratio predicted by Eq. (3) (Fig. 5, right).
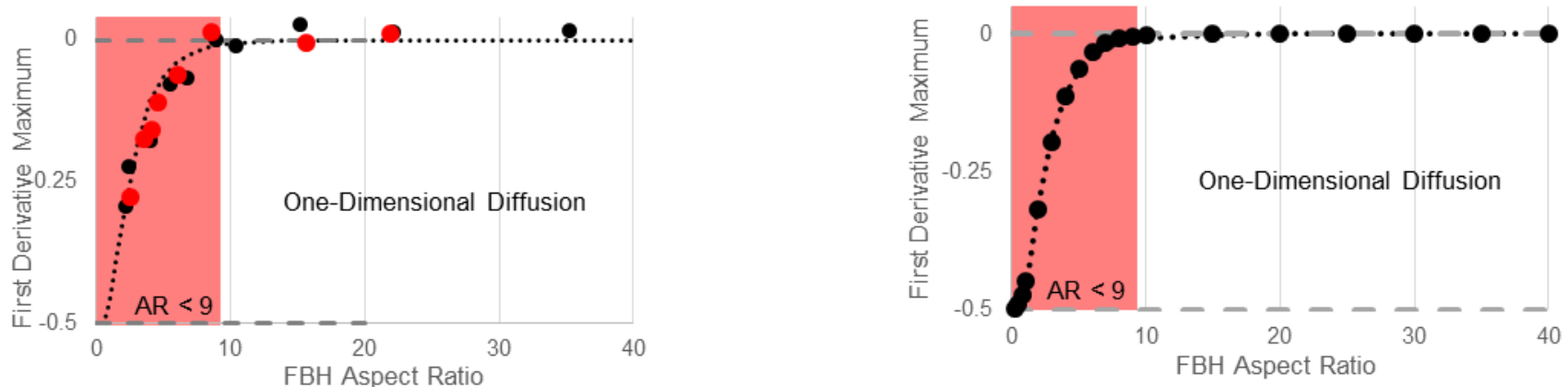

Fig. 5. Model (left) and experimental (right) first derivative maximum vs aspect ratio of FBH with aspect ratios described in Tables 1 (left), 2 (right, black) and 3 (right, red), along with the relationship described in Eq. (3) (dotted line).

The measured $t^{*}$ values for $\mathrm{FBH}$ in the low-aspect ratio regime occur earlier than those of the corresponding adiabatic slab, due to the signals' early divergence from the one-dimensional model. Depth measurements based on Eq. (2) will be shallower than the actual value, with the discrepancy increasing as aspect ratio decreases (Fig. 6). The shallowest holes in the stainless steel do not give accurate depth measurements due to under sampling and display significantly higher depth measurement errors than expected.

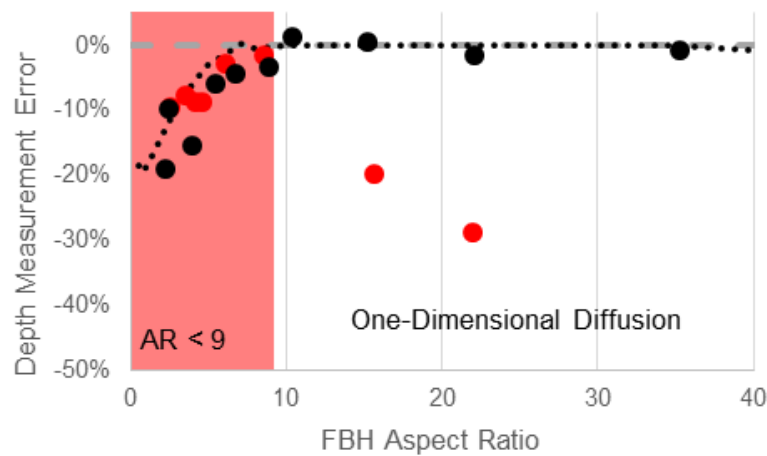

Fig. 6. Experimental depth measurement error for steel (black) and stainless steel (red) using the first derivative half maximum time (dots) compared to numerical modeling (dashed line).

When a first derivative signal reaches the adiabatic maximum of 0 , we can infer, based on the behavior of a single pixel at the center of the flaw, that the flaw is adiabatic. However, for lower maximum derivative amplitudes, the composition of the flaw becomes ambiguous; a low aspect ratio adiabatic flaw is not necessarily distinguishable from a larger aspect ratio inclusion with higher thermal effusivity that only partially obstructs the flow of heat. In such cases, three-dimensional analysis may be required for accurate depth measurement.

Viewed on a dimensionless scale, FBHs with equivalent aspect ratios behave the same, regardless of the host material in which they are contained. This provides an opportunity to better understand detectability with respect to flaw sizing across a broad range of materials. However, this discussion assumes that the host material is isotropic. Anisotropic materials, e.g. composites and polymers, initially behave isotropically when heat conduction is purely one-dimensional. However, once three-dimensional effects are introduced, the cooling pattern of anisotropic materials diverges from those discussed here. Such cooling patterns will be discussed in a separate paper.

In this discussion, we have addressed the range, based on aspect ratio, over which one-dimensional depth measurement techniques can be accurately performed. In principle, the aspect ratio limits we describe do not apply to detectability, since the raw contrast signal is proportional to the excitation energy applied to the sample. However, in practice, large excitation energies invoke additional problems (e.g. convection), and low aspect ratio features detected using 


\subsection{1/qirt.2016.005}

raw contrast with high excitation energy are spatially blurred compared to TSR processed images acquired in the early time regime.

\section{REFERENCES}

[1] Vavilov, V., "Infrared Techniques for Materials Analysis and Nondestructive Testing," Infrared Methodology and Technology, International Advances in NDT, X. Maldague, ed., Gordon and Breach, 230-309 (1994).

[2] Shepard, S.M and Maldague, X.P.V., "Infrared and Thermal Testing", in Aerospace NDT ASNT Industry Handbook, Ch. 11, edited by R. Bossi (ASNT, ( 2014).

[3] Shepard, S.M, "Flash thermography contrast model based on IR camera noise characteristics", Nondestructive Testing and Evaluation, 22(2-3), June 2007

[4] Ibarra-Castanedo, C. et al., "Comparative Study of Active Thermography Techniques for the Nondestructive Evaluation of Honeycomb Structures. Research", in Nondestructive Evaluation 20, 1-31 (2009).

[5] Balageas, D.L., "Defense and illustration of time-resolved pulsed thermography for NDE", in Quantitative InfraRed Thermography Journal 9(1), 3-32 (2012).

[6] Carslaw, H.S. and Jaeger, J.C., Conduction of Heat in Solids, Oxford University Press, Oxford, 1959.

[7] Shepard, S.M., Lhota, J.R., Rubadeux, B.A., Wang, D., and Ahmed, T., "Reconstruction and Enhancement of Active Thermographic Image Sequences," in Optical Engineering 42(5), 1337-1342 (2003).

[8] Shepard, S.M. and Frendberg Beemer, M, "Advances in Thermographic Signal Reconstruction", Proc. SPIE 9485, Thermosense: Thermal Infrared Applications XXXVII, 94850R, 2015.

[9] Parker, W.J., Jenkins, R.J., Butler, C.P., and Abbott, G.L., "A Flash Method of Determining Thermal Diffusivity, Heat Capacity, and Thermal Conductivity”, Journal of Applied Physics, 32 (9), 1961, pp. 1679-1684. 